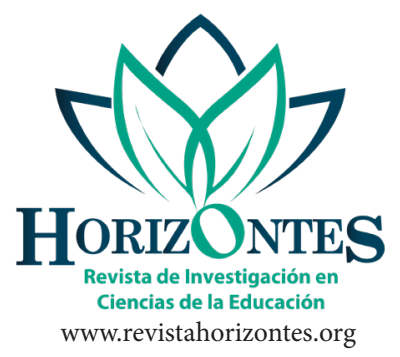

Horizontes. Revista de Investigación en Ciencias de la Educación Https://doi.org/10.33996/revistahorizontes.v6i22.318

Enero-marzo 2022

Volumen 6 / No. 22

ISSN: 2616-7964

ISSN-L: 2616-7964

pp. $101-107$

\title{
Calidad educativa en educación superior en tiempo de pandemia por el COVID -19
}

\author{
Educational quality in higher education in times of the COVID -19 pandemic \\ Qualidade educacional no ensino superior em tempos de pandemia COVID-19
}

ARTíCULO DE INVESTIGACIÓN

\section{Lidia Nelly Malpica Rodriguez \\ lmalpicar@ucvvirtual.edu.pe \\ https://orcid.org/0000-0003-2591-3546 \\ Universidad César Vallejo, Lima-Perú}

\author{
Teodoro Cruz Morales \\ cruzmo@ucvvirtual.edu.pe \\ https://orcid.org/0000-0002-4330-187X \\ Universidad César Vallejo, Lima-Perú
}

\author{
Eric Gálvez-Suárez \\ eric.galvez@upn.edu.pe \\ https://orcid.org/0000-0002-7642-5019
}

Universidad Privada del Norte, Trujillo-Perú

Artículo recibido 25 de octubre 2021, arbitrado y aceptado 11 de noviembre 2021 y publicado 28 de enero 2022

\section{RESUMEN}

El año 2020 la educación superior afrontó un cambio significativo al implementarse la educación virtual, debido a la pandemia originada por el COVID-19, a causa del confinamiento obligatorio, al no permitirse a las personas desplazarse implicó que, las clase fueran online de manera sincrónica o asincrónica, ante esta coyuntura surgieron cuestionamientos si este sistema educativo pudo ser el adecuado, formulándose como objetivo de estudio explorar la calidad educativa en educación superior en tiempo de pandemia por el COVID-19. Se empleó el enfoque cualitativo de revisión bibliográfica, la literatura consultada permitió develar dificultades comunes que limitaron brindar una educación de calidad: la falta de conectividad, dificultades en el manejo de los recursos tecnológicos y la carencia de competencias digitales.

Palabras clave: COVID-19; Educación superior; Pandemia; Virtualidad

\section{ABSTRACT}

In 2020, higher education faced a significant change when virtual education was implemented, due to the pandemic caused by COVID-19, due to mandatory confinement, by not allowing people to move, it implied that classes were synchronously online or asynchronous, at this juncture, questions arose as to whether this educational system could be adequate, formulating as a study objective to explore the educational quality in higher education in times of the COVID-19 pandemic. The qualitative approach of bibliographic review was used, the consulted literature revealed common difficulties that limited the provision of quality education: lack of connectivity, difficulties in managing technological resources and lack of digital skills.

Key words: COVID-19; Higher education; Pandemic; Virtuality

\section{RESUMO}

Em 2020, o ensino superior sofreu uma mudança significativa com a implantação do ensino virtual, devido à pandemia provocada pelo COVID-19, devido ao confinamento obrigatório, por não permitir a movimentação de pessoas, implicou que as aulas fossem online de forma síncrona ou assíncrona, tendo em vista Diante dessa situação, questionouse se esse sistema educacional poderia ser adequado, formulando-se como objetivo de estudo explorar a qualidade educacional no ensino superior em tempos de pandemia do COVID-19. Utilizou-se a abordagem qualitativa de revisão bibliográfica, a literatura consultada revelou dificuldades comuns que limitam a oferta de uma educação de qualidade: falta de conectividade, dificuldades na gestão dos recursos tecnológicos e falta de competências digitais.

Palavras-chave: COVID-19; Ensino superior; Pandemia; Virtualidade 


\section{INTRODUCCIÓN}

El año 2020 significó un cambio para el sistema educativo, debido a la aparición del COVID-19, un virus de fácil trasmisión por el contacto de persona apersona; determinó que la mayoría de los países del mundo implementaran medidas como fue el confinamiento obligatorio o cuarenta, por tal razón, se determinó que la educación fuera remota o a distancia en las instituciones de Educación Básica Regular (EBR) y Educación Básica alternativa (EBA); e la educación superior o técnico productiva se le denominó virtual.

La educación a distancia no era una novena en las universidades, (Martínez, 2008) este modelo educativo utilizado en universidades de Estados Unidos desde inicios del siglo XX, permitió mayor flexibilidad para los estudiantes; porque trasladó el espacio físico a los entornos virtuales, adecuando sus tiempos y estilos de aprendizaje, debido a que los recursos están a su disposición en el aula virtual, la cual se encuentra en una plataforma permite la interacción sincrónica o asincrónica (Valero et al., 2020).

La educación virtual estuvo permitida en Perú, principalmente en Posgrado y Formación para Adultos, Ley Universitaria $\mathrm{N}^{\circ} 30220$ (MINEDU, 2014) en el artículo 47, señaló que, tanto la educación presencial como la virtual deben tener los mismos estándares de calidad, sin embargo, en el contexto de la emergencia sanitaria se realizaron adecuaciones, para regularizar su implementación en el contexto de la pandemia, como contar con una plataforma que contenga todos los recursos necesarios, docentes competentes y estrategias de enseñanza aprendizaje concretas y entendibles.
En este contexto surgieron críticas, si este sistema educativo era el adecuado y/o de calidad, porque los estudiantes fueron obligados a recibirlo y no fue su elección; en esta línea resultó necesario explorar ¿cuál es el concepto de calidad? ¿De qué manera esta educación ayudaría a los estudiantes? ¿cómo serán los futuros profesionales formados en la virtualidad? no huno duda que los cuestionamientos fueron justificados, sin embargo, se intentó hacer lo posible para permitir fuera de calidad. Montenegro (2020) sostuvo que, mientras se formaron ciudadanos capaces de aportar a la sociedad desde su profesión, se podría hablar de una educación de calidad.

Tomando en consideración la Ley General de Educación $\mathrm{N}^{\circ} 28044$ (MINEDU, 2003) en el artículo 13 señaló que la calidad en educación, e obtiene cuando la persona logra el nivela idealizado en su formación, a su vez esto le permita ser mejores ciudadanos, el constructo calidad fue en un inicio ligado al tema empresarial y producción, (Lizarzaburu, 2016) e implica que tan bueno es un producto o servicio en función a las condiciones de satisfacción de los consumidores. Para Casassús (1995) citado por (Vaillant y Rodríguez, 2018) es un proceso que implica equidad, procesos y resultado; teniendo como indicadores de calidad los aprendizajes, personal docente, infraestructura y recursos.

En Perú la Ley Universitaria (MINEDU, 2014) asignó a la Superintendencia Nacional de Educación Superior Universitaria (SUNEDU), como entidad responsable de vigilar el aseguramiento a la calidad en la educación superior, la cual también estableció las Condiciones Básicas (MINEDU, 2019) de 
las cuales, son importantes en el contexto actual promover la investigación y formar ciudadanos capaces de aportar a la sociedad. Asimismo, el Proyecto Educativo Nacional (PEN) (CNE, 2019), la educación de calidad atiende a todos y todas por igual, bajo el principio de inclusión y equidad; propiciándose buenos aprendizajes. Por otro lado, la Política Nacional de Educación Superior y TécnicoProductiva (MINEDU, 2020) señaló entre sus objetivo, la necedad de promover el aseguramiento a la calidad mediante planes de mejora continua, además una institución fundamental perruna para certificar la calidad es el Sistema Nacional de Evaluación, Acreditación y Certificación de la Calidad Educativa (SINEACE) el cual fijó 34 estándares a considerar en la evaluación del servicio educativo los cuales permitirá la acreditación, estas serían las reformas en línea que estableció la Ley Universitaria (Sevillano, 2017), las dimensiones son: estratégica, integral, institucional y por resultados.

De acuerdo a lo antes expuesto, en Perú existe una normativa que permite vigilar se brinde una educación de calidad, por lo cual se formuló como objetivo de estudio explorar la calidad educativa en educación superior en tiempo de pandemia por el COVID-19.

\section{METODOLOGÍA}

Se empleó el enfoque cualitativo de revisión bibliográfica, el proceso de búsqueda se efectuó en ScienciDirect, Redalyc y Dialnet, entre las palabras clave encontradas se consideró: calidad, educación superior, COVID-19 y educación virtual, publicados desde el año 2019 al 2021. Según la perspectiva de este artículo conllevó a la recolección de información a través de posturas teóricas, conceptos y definiciones sobre la calidad educativa. Bajo este enfoque se definen tendencia, experiencias, posiciones y conclusiones, permitiendo interpretar desde diferentes realidades. El enfoque de este estudio implicó el acopio de información a través de posturas teóricas, conceptos y aportes sobre las categorías de la calidad educativa, las cuales permiten analizar el proceso de interpretación desde diferentes realidades. Para Uribe (2002) citado por (Guevara, 2016) la revisión documental tendrá cuatro etapas o fases: estrategia, técnica, procedimiento y actividad científica, los cuales se pusieron en práctica para la búsqueda de la literatura académica.

\section{DESARROLLO Y DISCUSIÓN}

La consulta de la literatura académica permitió establecer dos temas transversales, los cuales cuestionaron si la educación virtual durante la pandemia ocasionada por el COVID-19, era de calidad.

\section{Deficiencias en la conectividad y recursos tecnológicos}

Los estudiantes universitarios como se señaló líneas atrás fueron obligados a seguir la educación virtual, porque no había otra alternativa, la cual le permitía seguir con sus estudios, por tal razón gran parte de los estudiantes no contaba con los insumos necesario para seguir su formación profesional, los teléfonos móviles se convirtieron en el mecanismo que permitió seguir con sus clases, Chakraborty et al., (2021) señaló que, las universidades no estaban preparadas para esta situación, los autores estudiaron esta dificultad en la India, allí encontraron similitudes a las plataformas más empleadas la cuales fueron Clasroom y Blackboard. Para las videoconferencias Zoom y Meet, Para Guerrero et al., (2020) señalaron que, 
el empleo de las TIC, exigió el manejo de didáctica por parte de los docentes, (Castro et al., 2020) de acuerdo a la experiencia de una universidad de Buenos Aires, coinciden que adaptase y encontrar la forma adecuada fue un rápido proceso los cuales involucraron ensayos, colocaron como ejemplo que los docentes enviaron al principio PowerPoint con audios, los cuales demostraron no ser tan útiles, el nuevo sistema demostró que la educación estuvo afrontando un proceso de transformación; además sería apropiado considerar que estuvo generando una nueva forma de conocimiento.

Desde otra perspectiva García (2021) señaló que, un aspecto favorable para las universidades el cual permitió que los estudiantes se adaptaran más rápido, fue la edad, los jóvenes conocedores de redes sociales, pudieron ayudar en hacer de este sistema educativo más eficiente, sin embargo las distintas iniciativas académicas tuvieron que hacer frente a un problema mayor, la existencia de una brecha digital, deficiencias en conectividad, en Perú la Defensoria del Pueblo (2020) lanzó la alerta de estos problemas, los cuales ya se habían demostrado con cifras en los últimos censos, poco o nada habían hecho los distintos gobiernos, para implementar redes de conexión a internet a nivel nacional, (Gómez y Escobar, 2021) la situación en las zonas rurales, fue más complicada el acceso a la conectividad a diferencia de las urbanas, Pedró (2020) señaló que, en América latina solo el 52 $\%$ de los hogares contaban con las condiciones básicas necesarias como fueron Internet y recursos tecnológicos, por tal razón los distintos gobiernos debieron emplear recursos financieros, para atender esta brecha digital, la educación online es una alternativa; sin embargo resultó necesario diseñar estrategias para permitir la continuidad educativa, atendiendo principalmente a los más vulnerables (IIESAC, 2020).

\section{Las carencias de competencias digitales}

El implementar la educación virtual exigió a los docentes hacer uso de los recursos necesarios en los entornos virtuales, para os cuales muchos de ellos no estaban preparados, en el caso peruano, en aquellas universidades que se brandaba la educación virtual antes de la pandemia, los docentes tenía que acreditar mediante certificación contar con estas habilidades; sin embargo lo repentino de esta coyuntura implicó que la inducción fuera de manera acelerada, para los docentes de universidades públicas colocó a su disposición la plataforma "Conectados", con tutoriales y recursos que fortalezcan su práctica pedagógica. La forma de enseñanza implicó se cuestionará si los aprendizajes fueron de calidad; las principales preocupaciones fueron aquellas carrearas las cuales necesitaban interactuar o el empleo de talleres. Ortega et al., (2021) manifiesta que los docentes consiguieron adaptarse, empleando diversas estrategias metodológicas con el afán de mejorar los aprendizajes de los estudiantes y brindarles una adecuada retroalimentación.

Un aspecto preocupante de las deficiencias en el empleo de las competencias digitales, fueron las afecciones emocionales en los docentes que afrontaron situaciones de estrés (Apaza et al., 2020) las cuales se manifestaron en insomnio, depresión irritabilidad, etc. Porque a los temas laborales de trasladar sus actividades profesionales a su hogar, se sumó la angustia e incertidumbre generada por la pandemia (Broche Pérez et al., 2020), También fue un obstáculo el no tener un espacio apropiado para 
su desempeño laboral, las familias compartieron meses de encierro interrumpido, el compartir un ambiente por tiempo prolongado no adecuado contribuyó en la frustración para poder desarrollar adecamente su trabajo (Ruperti-Lucero et al., 2021).

La educación superior afrontó problemas financieros, principalmente las de gestión privada porque un importante número de estudiantes debieron abandonar sus estudios por las crisis económica, algunas optaron por juntar mayor número de estudiantes en la aulas virtuales, complicando el desempeño laboral de los catedráticos, por tal motivo se cuestionó que la falta de recursos financiero pudieran bajar la calidad educativa (Rogers y Sabarwal, 2020).

\section{CONCLUSIÓN}

La emergencia sanitaria ocasionada por el COVID-19 determinó que la educación superior fuera virtual, esta modalidad ya era empelada por algunas casas de estudio en las escuelas de Posgrado y Formación para Adultos, porque permitió flexibilidad, teniendo claro que antes de la pandemia fue una elección; sin embargo pro la emergencia sanitaria fue una obligación, que determinó los cuestionamientos, si este tipo de educación era de calidad, dada esta circunstancias las instituciones vigilantes para el cumplimiento de un adecuado servicio educativo como la SUNEDU y el SINEACE, retuvieron que generar adecuaciones para vigilar si el nuevo sistema fue de calidad.

De acuerdo a la Ley universitaria, la calidad se expresa en la formación de profesionales útiles para la sociedad, en tal sentido fue cuestionado si este modelo educativo permitió brindar experiencias de aprendizaje de calidad.
La revisión de la literatura permitió erguir dos dificultades frecuentes que pudieron limitar que la educación virtual fura de calidad, como fuero las brechas de digitales, los estudiantes tuvieron dificultades para conectarse por internet para aprender sus clase; asimismo la falta de recursos tecnológico, lo más común fue el empleo de los teléfonos celulares para seguir sus clases, otro aspecto que en la literatura se encontró coincidencias fue las debilidades en competencias digitales, la frustración de o poderse adaptar generó estrés que pudo afectar a los docentes y por consiguiente su práctica profesionales.

Fielmente, los cuestionamientos si esta educación es de calidad, se pudo inferir que se fortaleció creándose nuevas estrategias y medos, porque este sistema educativo de acuerdo a la nueva normalidad perdura por mucho tiempo.

\section{REFERENCIAS}

Apaza P., C. M., Seminario Sanz, R. S., y Santa-Cruz Arévalo, J. E. (2020). Factores psicosociales durante el confinamiento por el covid-19 - perú. Revista Venezolana de Gerencia, 25(90), 402413. https://doi.org/10.37960/rvg.v25i90.32385

Broche Pérez, Y., Fernández Castillo, E., y Reyes Luzardo, D. A. (2020). Consecuencias psicológicas de la cuarentena y el aislamiento social durante la pandemia de COVID-19. Revista Cubana de Salud Pública, 46, 1-14. https://orcid.org/0000-0002-0613-3459

Castro, M., Paz, M., y Cela, E. (2020). Aprendiendo a enseñar en tiempos de pandemia COVID-19: nuestra experiencia en una universidad pública de Argentina. Revista Digital de Investigación En Docencia Universitaria, 14(2). https://doi. org/10.19083/ridu.2020.1271

Chakraborty, P., Mittal, P., Gupta, M. S., Yadav, S., y Arora, A. (2021). Opinion of students on online education during the COVID-19 pandemic. In 
Human Behavior and Emerging Technologies (Vol. 3, Issue 3, pp. 357-365). https://doi. org/10.1002/hbe2.240

CNE. (2019). Proyecto Educativo Nacional al 2036. In Todos somos educadores (Issue 44)

Defensoría del Pueblo. (2020). Educacion En Pandemia. https://www.defensoria.gob.pe/ wp-content/uploads/2020/08/Serie-InformesEspeciales-No-027-2020-DP-La-educaciónfrente-a-la-emergencia-sanitaria.pdf

García, L. (2021). COVID-19 y educación a distancia digital_ preconfinamiento, confinamiento $y$ posconfinamiento.pdf. http://revistas.uned.es/ index.php/ried/article/view/28080/21886

Gómez A, I., y Escobar M, F. (2021). Educación virtual en tiempos de pandemia: Incremento de la desigualdad social en el Perú. Biblioteca Electrónica Científica En Línea, I, 1-13. https:// preprints.scielo.org/index.php/scielo/preprint/ view/1996\#.YFvtuoYYVe0.mendeley

Guerrero Jirón, J. R., Vite Cevallos, H. A., y Feijoo Valarezo, J. M. (2020). Uso de la tecnología de información y comunicación y las tecnologías de aprendizaje y conocimiento en tiempos de Covid-19 en la Educación Superior. Conrado, 16(77), 338-345.

Guevara, R.-. (2016). El estado del arte en la investigación : $i$ análisis de los conocimientos acumulados o indagación por nuevos sentidos? The State of the Art as a Research Technique: Redalyc.Org, 16

IIESAC. (2020). COVID-19 y educación superior: De los efectos inmediatos al día después. Unesco, 57. http://www.iesalc.unesco.org/wp-content/ uploads/2020/04/COVID-19-060420-ES-2.pdf

Lizarzaburu Bolaños, E. R., E. R. (2016). La gestión de la calidad en Perú: un estudio de la norma ISO 9001, sus beneficios y los principales cambios en la versión 2015. Universidad \& Empresa, 18(30), 33-54. https://doi.org/10.12804/rev. univ.empresa.30.2016.02

Martínez, C. (2008). La educación a distancia: sus características y necesidad en la educación actual. Educación, 17(33), 1. http://revistas. pucp.edu.pe/index.php/educacion/article/ view/1532/1477
MINEDU. (2014). Ley Universitaria No 30220. Ley, 54, 68. http://www.minedu.gob.pe/reformauniversitaria/pdf/ley_universitaria.pdf

MINEDU. (2017). Ley General de Educación $\mathrm{N}^{\circ}$ 28044. Sobre Educación, 1-36. http:// www.minedu.gob.pe/p/ley_general_de_ educacion_28044.pdf

MINEDU. (2020). Política Nacional de Educaión Superior y Técnico Productiva

Montenegro, O. J. (2020). La calidad en la docencia universitaria. Una aproximación desde la percepción de los estudiantes. Educación, 29(56), 116-145 http://www.scielo.org.pe/ scielo.php?script $=$ sci_arttext \&pid $=$ S101994032020000100116\&lang=es\%0Ahttp://www. scielo.org.pe/pdf/educ/v29n56/2304-4322educ-29-56-116.pdf

Ortega Ortigoza, D., Rodríguez Rodríguez, J., y Mateos Inchaurrondo, A. (2021). Educación superior y la COVID-19: adaptación metodológica y evaluación online en dos universidades de Barcelona. Revista Digital de Investigación En Docencia Universitaria, 15(1), e1275. https://doi.org/10.19083/10.19083/ ridu.2021.1275

Pedró, F. (2020). COVID-19 y educación superior en América Latina y el Caribe: Efectos, impactos y recomendaciones políticas. Análisis Carolina, 1-15. https://doi.org/10.33960/ac_36.2020

Rogers, H., y Sabarwal, S. (2020). Covid-19: Impacto En La Educacion Y Respuestas De Política Pública. Reporte Del Banco Mundial, 1-56. http://documents1.worldbank.org/ curated/en/804001590734163932/pdf/TheCOVID-19-Pandemic-Shocks-to-Educationand-Policy-Responses.pdf

Ruperti-Lucero, E. M., Espinel-Guadalupe, J. V., Naranjo-Cabrera, C. R., y Aguilar-Pita, D. V. (2021). Conciliación de la vida familiar y bienestar laboral, análisis de roles sociales y género en tiempos de Covid-19. Caso Ecuador. Universidad Estatal de Milagro, 1-9. https:// chakinan.unach.edu.ec/index.php/chakinan/ article/view/525/495 
Sevillano, S. (2017). El sistema de acreditación universitaria en el Perú: marco legal y experiencias recientes. Revista de Educación $y$ Derecho, 0(15). https://doi.org/10.1344/ REYD2017.15.20952

SINEACE. (2016). Modelo SINEACE. (2016). Modelo de Acreditación para Programas de Estudios de Educación Superior Universitaria. Sineace, 36.

Vaillant, D., y Rodríguez, E. (2018). Perspectivas de UNESCO y la OEI sobre la calidad de la educación. Monarca, H. Calidad de La Educación En Iberoamérica: Discursos, Políticas y Prácticas, December, 136-154. https://ie.ort. edu.uy/innovaportal/file/73018/1/perspectivasde-unesco-y-oei-vaillant-rodriguez.pdf

Valero, N. J., Castillo, A. L., Rodríguez, R., Padilla, M., y Cabrera, M. (2020). Retos de la educación virtual en el proceso enseñanza aprendizaje durante la pandemia de Covid-19. Dominio de Las Ciencias, 6(4), 1201-1220 